Nota Científica

\title{
Presencia de Monosteira unicostata (Mulsant y Rey, 1852) (Hemiptera: Tingidae) en Chile
}

Presence of Monosteira unicostata (Mulsant and Rey, 1852) (Hemiptera: Tingidae) in Chile

$$
\text { Juan Francisco Campodonico }{ }^{1,2}{ }^{(\mathbb{D}} \text {, Andrés Fierro }{ }^{3}{ }^{\mathbb{R}} \text { y Sergio Rothmann }{ }^{4}
$$

${ }^{1}$ Programa de Doctorado en Ciencias mención Ecología y Evolución, Escuela de Graduados, Facultad de Ciencias, Universidad Austral de Chile. Av. Rector Eduardo Morales Miranda s/n, Edificio Emilio Pugín, Valdivia, Región de los Ríos, Chile. 隐 juan.campodonico@ug.uchile.cl ${ }^{2}$ Instituto de Ciencias Ambientales y Evolutivas, Facultad de Ciencias, Universidad Austral de Chile. ${ }^{3}$ Laboratorio de Ecología y Conservación (LEC), Departamento de Gestión Agraria, Universidad de Santiago de Chile, Av. Libertador Bernardo O'Higgins 3363, Estación Central, Santiago, Chile. ${ }^{4}$ Servicio Agrícola y Ganadero, Unidad de Entomologí, Subdepartamento de Laboratorios y Estación Cuarentenaria Agrícola, Pudahuel, Santiago, Chile.

\section{ZooBank: urn:lsid:zoobank.org:pub:38CE98CD-2BD2-4B51-9841-A5AD6DB45334 https: / / doi.org/10.35249/ rche.47.1.21.10}

Resumen. Se registra la presencia de Monosteira unicostata (Mulsant y Rey, 1852) en la zona central de Chile, siendo el segundo país de Sudamérica en el que se detecta después de Argentina. Este chinche es originario de la Región Paleártica y es perjudicial para el cultivo de diversas especies frutales.

Palabras clave: Chinche; especie exótica; faunística; Heteroptera; plaga.

Abstract. The presence of Monosteira unicostata (Mulsant and Rey, 1852) is reported for the central zone of Chile. Therefore Chile becomes the second country in South America, after Argentina, where this species is detected. It is originary from the Palearctic Region and is harmful to the cultivation of several fruit trees.

Key words: Alien species; faunistics; Heteroptera; pest; true bug.

En la última década ha habido un importante incremento en el número de especies exóticas de chinches (Hemiptera: Heteroptera) que se han introducido y establecido exitosamente en Chile (Faúndez y Rider 2017; Faúndez et al. 2017, 2018, 2020a; Flores y Riveras 2018). Dada la dieta fitófaga de buena parte de sus integrantes, existen plagas de heterópteros importantes para diversos cultivos agrícolas como Bagrada hilaris (Burmeister, 1835) y Halyomorpha halys (Stål, 1855) (Pentatomidae) (Faúndez et al. 2016; Faúndez y Rider 2017). También existen especies que se pueden considerar de importancia silvícola, como Leptoglossus occidentalis Heidemann, 1910 (Coreidae) y Boisea trivittata (Say, 1825) (Rhopalidae) (Faúndez y Rocca 2017; Faúndez et al. 2020a). Algunas de estas especies llegan a causar molestias directas a seres humanos, tanto por agregaciones en casas como por picaduras ocasionales (Faúndez 2018; Faúndez et al. 2020b).

En la presente contribución se da a conocer la presencia del “tigre del almendro" Monosteira unicostata (Mulsant y Rey, 1852) (Tingidae: Tinginae), cuyos ejemplares fueron determinados

Recibido 10 Agosto 2020 / Aceptado 20 Octubre 2020 / Publicado online 26 Febrero 2021

Editor Responsable: José Mondaca E. 
mediante la descripción de Scudder (2013). Esta especie resulta perjudicial para el cultivo del almendro (Prunus dulcis (Mill.) D.A. Webb) y otros frutales de la familia Rosaceae, además de nogales (Juglans spp.; Juglandaceae). También se suele encontrar frecuentemente asociada a salicáceas, particularmente álamos (Populus spp.) y sauces (Salix spp.) (Stonedahl et al. 1992; Carpintero et al. 2017), especies arbóreas exóticas cultivadas frecuente en Chile, lo que puede suponer una amenaza al facilitar la propagación de este insecto al resto del país.

Monosteira unicostata (Fig. 1) ha sido detectada por el Servicio Agrícola y Ganadero de Chile desde el 2018 en la Región Metropolitana de Santiago, producto de las actividades de vigilancia agrícola y forestal implementadas en el país, estando presente actualmente entre las regiones Metropolitana y Biobío (base de datos SAG 2019). Por los registros obtenidos durante 2019 y 2020, sumado a la dificultad para erradicar las poblaciones de esta plaga, y por su frecuencia y capacidad para alimentarse de diversas plantas cultivadas, esta especie se puede considerar como establecida en el territorio chileno.
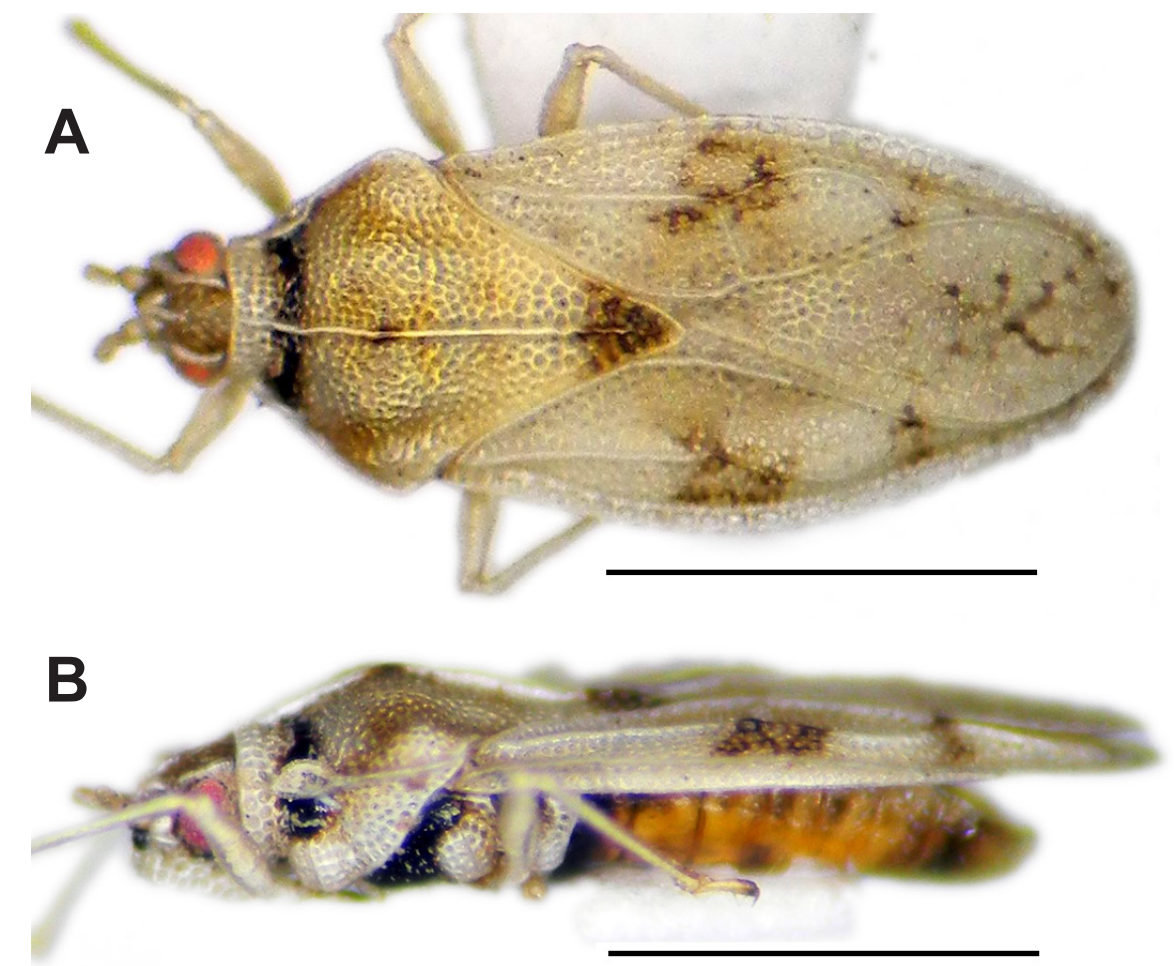

Figura 1. Monosteira unicostata (Mulsant y Rey), hembra. A. Vista dorsal. B. Vista lateral. Escalas: $1 \mathrm{~mm}$. / Monosteira unicostata (Mulsant and Rey), female. A. Dorsal View. B. Lateral View. Scale bars: 1 mm.

Material examinado. Chile, Región Metropolitana de Santiago: San Bernardo, camino El Romeral, E 339369 N 6270904 WGS84 Huso 19, Ex Populus alba, 8.I.2018, P. Bosch leg., 3 hembras, 1 macho; Colina, camino Santa Luisa, E 342702 N 6317736 WGS84 Huso 19, Ex Cupressus sp., 31.I.2018, E. Zúñiga leg., 1 hembra, 1 macho; Colina, San Vicente de Lo Arcaya, E 342821 N 6318363 WGS84 Huso 19, Ex, Salix babilonica, 31.I.2018, E. Zúñiga leg., 14 hembras, 30 machos, 8 ninfas; Colina, Ex Jacaranda mimosifolia, 03.I.2019, C. Cortés leg., 1 macho; Pudahuel, Ex Quercus sp., 10.I.2019, M. Bustos leg., 1 hembra; Planta CMPC Laja, Prov. Biobío, trampa funnel, 09 Octubre 2020, leg. M. Beéche, 6 hembras, 4 machos (todos los anteriores depositados en la colección de la Unidad de Entomología, Complejo Lo Aguirre, Servicio Agrícola y Ganadero, Santiago, Chile); Provincia de Chacabuco, Huechún, 3305'S 7045’O, X.2019, A. Fierro leg., 1 hembra (colección particular Andrés Fierro). 
En el nuevo mundo esta especie ha sido registrada en Canadá y Argentina (Scudder 2013; Carpintero et al. 2017). La presencia en Argentina, y en particular en Mendoza, da indicios de su posible ruta de ingreso a través de medios de transporte terrestre que llegan a la zona central de Chile, situación similar a la propuesta anteriormente para Rhytidodus decimusquartus (Schrank, 1776) (Hemiptera: Cicadellidae), especie con un patrón de invasión similar al de M. unicostata (Campodonico 2015).

En Chile la fauna de Tingidae se encuentra representada por siete especies agrupadas en los géneros Coleopterodes Philippi, 1864, Corythaica Stål, 1873, Corythucha Stål, 1873, Teleonemia Costa, 1864 (Tinginae) y Stenocader Drake y Hambleton, 1934 (Cantacaderinae) (Prado 1990, 2008; Montemayor y Coscarón 2005; Carpintero y Montemayor 2008; Faúndez y Carvajal 2014; Faúndez y Lüer 2015; Elgueta et al. 2018). Monosteira unicostata se distingue del resto de los tíngidos presentes en Chile por carecer de expansiones paranotales y caperuza (presentes en Corythaica y Corythucha), y presentar el pronoto con expansión posterior (ausente en Stenocader) y una sola carena en el disco (a diferencia de tres en Coleopterodes y Teleonemia). Se debe destacar también la presencia en Chile de la familia Piesmatidae, representada por Miespa reedi (Drake, 1939) (Drake 1939; Kment 2019). Estos pueden llegar a confundirse con los Tingidae debido a que también poseen la característica textura superficial en forma de encaje. Sin embargo, se diferencian por la presencia de tricobotrios abdominales, de los que los tingidos carecen (Guidoti et al. 2015).

\section{Agradecimientos}

JFC es beneficiario de la beca ANID-PFCHA de Doctorado Nacional 2019-21190918.

\section{Literatura Citada}

Campodonico, J.F. (2015) On the presence of Rhytidodus decimusquartus (Schrank, 1776) (Hemiptera: Cicadellidae: Idiocerinae) in Chile. Arquivos Entomolóxicos, 14: 261-263.

Carpintero, D.L., López Plantey, R.J., Quiroga, V.N. y Holgado, M.G. (2017) Primera cita de Monosteira unicostata (Mulsant \& Rey, 1852) (Hemiptera: Tingidae) para la República Argentina, encontrada en la Provincia de Mendoza. Historia Natural (Tercera Serie), 7(1): 121-127.

Carpintero, D.L. y Montemayor, S.I. (2008) Revision of the Cantacaderinae (Heteroptera, Tingidae) from Argentina and Chile, with the description of a new species of Cantacaderini. Deutsche Entomologische Zeitschrift, 55(1): 109-116.

Drake, C.J. (1939) Chilean Tingitoidea (Hemiptera). Revista de Entomología, 10: 330-334.

Elgueta, M., Faúndez, E.I. y Campodonico, J.F. (2018) Orden Hemiptera. (pp. 292-297). En: MMA. Biodiversidad de Chile: Patrimonio y Desafíos. Tercera edición. Tomo I. Ministerio del Medio Ambiente, Santiago, Chile.

Faúndez, E.I. (2018) From agricultural to household pest: The case of the painted bug Bagrada hilaris (Burmeister) (Heteroptera: Pentatomidae) in Chile. Journal of Medical Entomology, 55(5): 1365-1368.

Faúndez, E.I. y Carvajal, M.A. (2014) A new hostplant record for Coleopterodes liliputianum (Signoret, 1864) (Hemiptera: Heteroptera: Tingidae), with comments on its distribution in Chile. Boletín de Biodiversidad de Chile, 9: 42-45.

Faúndez, E.I., Carvajal, M.A. y Sarmiento, C. (2020a) Detection of the boxelder bug Boisea trivittata (Say, 1825) (Heteroptera: Rhopalidae) in Chile. Heteroptera Poloniae - Acta Faunistica, 14: 125-128.

Faúndez, E.I., Carvajal, M.A. y Villablanca, J. (2020b) Alien invasion: The case of the Western Conifer-Seed bug (Heteroptera: Coreidae) in Chile, overreaction, and misidentifications. Journal of Medical Entomology, 57(1): 297-303. 
Faúndez, E.I. y Lüer, A. (2015) Primer registro de Stenocader mapu Carpintero \& Montemayor, 2008 (Heteroptera: Tingidae) en Chile. Anales del Instituto de la Patagonia, 43(1): 163-164.

Faúndez, E.I., Lüer, A. y Cuevas, Á.G. (2017) The establishment of Bagrada hilaris (Burmeister, 1835) (Heteroptera: Pentatomidae) in Chile, an avoidable situation?. Arquivos Entomolóxicos, 17: 239-241.

Faúndez, E.I., Lüer, A., Cuevas, Á.G., Rider, D.A. y Valdebenito, P. (2016) First record of the painted bug Bagrada hilaris (Burmeister, 1835) (Heteroptera: Pentatomidae) in South America. Arquivos Entomolóxicos, 16: 175-179.

Faúndez, E.I. y Rider, D.A. (2017) The brown marmorated stink bug Halyomorpha halys (Stål, 1855) (Heteroptera: Pentatomidae) in Chile. Arquivos Entomolóxicos, 17: 305-307.

Faúndez, E.I. y Rocca, J.R. (2017) La chinche de las coníferas occidental, Leptoglossus occidentalis Heidemann (Heteroptera: Coreidae) en Chile; rápida expansión, posibles impactos y desafíos. Revista Chilena de Entomología, 42: 25-27.

Faúndez, E.I., Rocca, J. y Villablanca, J. (2018) On the establishment of the western conifer seed bug Leptoglossus occidentalis Heidemann (Heteroptera: Coreidae) in Chile. Revista Chilena de Entomología, 44(2): 207-210.

Flores, A. y Riveras, M. (2018) Primer registro de Metopoplax ditomoides (Costa) (Hemiptera: Oxycarenidae) en Chile. Revista Chilena de Entomología, 44(1): 35-39.

Guidoti, M., Montemayor, S.I. y Guilbert, É. (2015) Lace bugs (Tingidae). In: Panizzi, A.R. y Grazia, J. (Eds.) True bugs (Heteroptera) of the Neotropics (pp. 395-419). Springer, Dordrecht.

Kment, P. (2019) Miespa reedi (Drake, 1939) (Hemiptera: Heteroptera: Piesmatidae) - first record from Argentina. Heteroptera Poloniae - Acta Faunistica, 13: 101-104.

Montemayor, S.I. y Coscarón, M.C. (2005) Taxonomic revision of Coleopterodes Philippi (Heteroptera: Tingidae). Zootaxa, 1092(1): 31-38.

Prado, C.E. (1990) Presencia en Chile de Corythucha ciliata (Say) (Hemiptera: Heteroptera: Tingidae). Revista Chilena de Entomología, 18: 53-55.

Prado, E. (2008) Conocimiento actual de Hemiptera-Heteroptera de Chile con lista de especies. Boletín del Museo Nacional de Historia Natural, 57: 31-75.

Servicio Agrícola y Ganadero (2019) Registros de Plagas. Subdepartamentos de Vigilancia y Control de Plagas: Agrícolas y Forestales. Depto. Sanidad Vegetal. División Protección Agrícola y Forestal. Servicio Agrícola y Ganadero. Chile.

Scudder, G.G.E. (2013) Monosteira unicostata (Mulsant \& Rey) (Hemiptera: Tingidae) established in North America, with a key to the genera of Tingidae in Canada. Entomologica Americana, 118(1): 295-297.

Stonedahl, G.M., Dolling, W.R. y Duheaume, G.J. (1992) Identification guide to common tingid pests of the world (Heteroptera: Tingidae). International Journal of Pest Management, 38(4): 438-449. 